\title{
Oncogenic KIT mutations induce STAT3- dependent autophagy to support cell proliferation in acute myeloid leukemia
}

Clément Larrue ${ }^{1,2}$, Quentin Heydt ${ }^{1,2}$, Estelle Saland ${ }^{1,2}$, Héléna Boutzen ${ }^{1,2}$, Tony Kaoma ${ }^{3}$, Jean-Emmanuel Sarry ${ }^{1,2}$, Carine Joffre $\mathbb{1}^{1,2}$ and Christian Récher (10)

\begin{abstract}
Autophagy is associated with both survival and cell death in myeloid malignancies. Therefore, deciphering its role in different genetically defined subtypes of acute myeloid leukemia (AML) is critical. Activating mutations of the KIT receptor tyrosine kinase are frequently detected in core-binding factor AML and are associated with a greater risk of relapse. Herein, we report that basal autophagy was significantly increased by the KIT ${ }^{\mathrm{D} 16 \mathrm{~V}}$ mutation in AML cells and contributed to support their cell proliferation and survival. Invalidation of the key autophagy protein Atg12 strongly reduced tumor burden and improved survival of immunocompromised NSG mice engrafted with KIT ${ }^{\mathrm{D} 816 \mathrm{~V}}$ TF-1 cells. Downstream of KITD816V , STAT3, but not AKT or ERK pathways, was identified as a major regulator of autophagy. Accordingly, STAT3 pharmacological inhibition or downregulation inhibited autophagy and reduced tumor growth both in vitro and in vivo. Taken together, our results support the notion that targeting autophagy or STAT3 opens up an exploratory pathway for finding new therapeutic opportunities for patients with CBF-AML or others malignancies with $K I T^{\mathrm{D} 816 \mathrm{~V}}$ mutations.
\end{abstract}

\section{Introduction}

Autophagy is an adaptive and protective cellular program activated during nutrient deprivation, growth factor withdrawal, or metabolic stress to maintain cellular homeostasis and recycle damaged organelles ${ }^{1}$. This dynamic process involves the rearrangement of subcellular membranes in order to sequester organelles or long-lived proteins and to then release these contents into the lysosomal machinery for degradation and recycling.

Defects in autophagy are associated with various diseases including cancer ${ }^{2}$. The role of autophagy in cancer is complex and depends on the tumor subtype, the stage of tumor progression, cellular context, and/or the drugs that

Correspondence: Christian Récher (recher.christian@iuct-oncopole.fr)

${ }^{1}$ Cancer Research Center of Toulouse (CRCT), UMR1037 INSERM, ERL5294 CNRS, Equipe Labellisée LIGUE, Toulouse, France

${ }^{2}$ University of Toulouse, Toulouse, France

Full list of author information is available at the end of the article.

These authors contributed equally: Carine Joffre, Christian Récher induce this process ${ }^{3,4}$. Indeed, autophagy may suppress cancer initiation ${ }^{5,6}$ while enabling cell survival and growth of aggressive cancers, particularly during chemotherapeutic stress ${ }^{7}$. Oncogenes, such as mutant $\mathrm{Kras}^{\mathrm{G} 12 \mathrm{D}}$ or $B r a f^{\mathrm{V} 600 \mathrm{E}}$, have a high basal autophagy and need autophagy for growth ${ }^{8,9}$. In myeloid malignancies, BCR-ABL leukemic cells are also dependent on autophagy for cell survival and leukemogenesis ${ }^{10,11}$. Pharmacological inhibition of autophagy to potentiate BCR-ABL inhibitors is currently being investigated in chronic myeloid leukemia ${ }^{12}$.

It has recently been proposed that autophagy is required for leukemic development, as invalidation of $\operatorname{Atg} 7$ or $\operatorname{Atg} 5$ in a murine $M l l-E n l$ leukemic model impaired tumor growth $^{13}$ and involved in resistance to chemotherapy ${ }^{13,14}$. Moreover, high levels of autophagy seem to be associated with poor prognosis in acute myeloid leukemia (AML) patients. Indeed, AML cells from patients with complex cytogenetic abnormalities vs. normal karyotype or

\section{(c) The Author(s) 2019}

(c) (i) Open Access This article is licensed under a Creative Commons Attribution 4.0 International License, which permits use, sharing, adaptation, distribution and reproduction cc) in any medium or format, as long as you give appropriate credit to the original author(s) and the source, provide a link to the Creative Commons license, and indicate if changes were made. The images or other third party material in this article are included in the article's Creative Commons license, unless indicated otherwise in a credit line to the material. If material is not included in the article's Creative Commons license and your intended use is not permitted by statutory regulation or exceeds the permitted use, you will need to obtain permission directly from the copyright holder. To view a copy of this license, visit http://creativecommons.org/licenses/by/4.0/. 
classified in adverse-risk group compared with the intermediate- or favorable-risk AML groups display higher levels of autophagy ${ }^{15}$. Accordingly, the mutant receptor tyrosine FLT3-ITD, which is also associated with a poor outcome, supports an elevated autophagy flux ${ }^{16}$. In addition, autophagy represents a mechanism of chemoresistance in specific AML models. This is the case upon mTORC1/mTORC2 inhibitors ${ }^{17}$ or upon histone deacetylase inhibitors ${ }^{18}$. However, in other contexts, inducing autophagy could be detrimental to leukemic cells. For instance, autophagy induced by arsenic trioxide, all-trans retinoic acid, or bortezomib contributes to cell death through the degradation of oncoproteins such as PML-RARA or FLT3-ITD in AML cells ${ }^{19,20}$. Thus, elucidating the role of autophagy in genetically defined AML subtypes is critical.

KIT mutations are found in $20-40 \%$ of patients with core-binding factors (CBFs) AML. These include AML with a $\mathrm{t}(8 ; 21)(\mathrm{q} 22 ; \mathrm{q} 22)$ or inv $^{16}(\mathrm{p} 13 \mathrm{q} 22)$ chromosomal rearrangement, which generate RUNX1T1-RUNX1 and $C B F b / M Y H I I$ fusion genes ${ }^{21}$. These mutations are associated with higher incidences of relapse after intensive chemotherapy and are associated with a poor prognosis ${ }^{22}$. The most frequent KIT mutations are point mutations, insertions, or deletions in exons 8 and 17, which encode the activation loop in the kinase domain and an extracellular region of KIT, respectively. Mutated KIT induces constitutive activation of phosphoinositide 3-kinase (PI3K)/AKT, ERK, and STAT3 pathways, and cooperates with RUNX1T1-RUNX1 to induce AML in mice ${ }^{23}$. As these cell signaling pathways interfere with autophagy, we herein report on our investigation into the role of autophagy in KIT-mutated AML cells.

\section{Results \\ KIT mutations induce autophagy, which supports cell proliferation and survival in AML cells}

We first compared basal autophagy in a TF-1 leukemic cell line that constitutively expressed wild-type $K I T$ and in TF-1 engineered to express a KIT-D816V mutant (TF-1 $\mathrm{KIT}^{\mathrm{D} 816 \mathrm{~V}}$ ). During autophagy, the microtubule-associated protein-1 light chain 3 (LC3-I) is converted to membranebound LC3-II and specifically associates with autophagosomes ${ }^{24}$. In order to address autophagic flux in cells harboring a $K I T^{\mathrm{D} 816 \mathrm{~V}}$ mutation, cells were incubated with lysosomal protease inhibitors, chloroquine, or Bafilomycin $\mathrm{A}$, and then stained with cyto-ID or processed using western blotting analysis and LC3-I/II expression. Upon treatment of these autophagy inhibitors, both methods showed increased cyto-ID staining or LC3-II expression, which was more pronounced in TF- 1 KIT ${ }^{\mathrm{D} 816 \mathrm{~V}}$ compared with TF-1 cells and was consistent with active autophagic flux rather than a defect in autophagosome-lysosome fusion (Fig. 1a, b). Moreover, immunofluorescence microscopy showed accumulation of LC3-positive structures that was significantly enriched in TF-1 KIT ${ }^{\mathrm{D} 816 \mathrm{~V}}$ compared with TF-1 cells (Fig. 1c, d). $\mathrm{KIT}^{\mathrm{D} 816 \mathrm{~V}}$ was then pharmacologically inhibited by PKC412 (midostaurin) or was genetically invalidated by inducible short hairpin RNA (shRNA). Both methods demonstrated that targeting $\mathrm{KIT}^{\mathrm{D} 816 \mathrm{~V}}$ strongly decreased autophagic flux (Fig. 1e, f).

To further investigate the role of autophagy in this model, we transduced TF-1 and TF-1 KIT ${ }^{\mathrm{D} 816 \mathrm{~V}}$ with lentiviral vectors encoding inducible shRNA against Atg12 or Vps34, two key autophagic proteins. As expected, downregulation of Atg12 and Vps34 by shRNAs was associated with inhibition of $\mathrm{KIT}^{\mathrm{D} 816 \mathrm{~V}}$-induced autophagy as shown by decreased LC3-II accumulation or LC3positive structures (Supplementary Fig. S1A-C). Moreover, we showed that the pharmacological inhibition of KIT by PKC412 significantly reduced the proliferation of $\mathrm{KIT}^{\mathrm{D} 816 \mathrm{~V}}$ expressing TF-1 cells without impacting the TF1 cells number (Fig. 2a). This result indicates that the mutated cell line is dependent on KIT activation to proliferate. We then investigated the functional consequence of $\mathrm{KIT}^{\mathrm{D} 816 \mathrm{~V}}$-induced autophagy on $\mathrm{KIT}^{\mathrm{D} 816 \mathrm{~V}}$-dependent proliferation and survival by comparing the impact of Atg12 and Vps34 depletion in TF-1 and TF-1 KIT ${ }^{\text {D816V }}$ cell lines. Interestingly, although the invalidation of Atg12 and Vps34 had a small impact on TF-1 cell proliferation, significant inhibition of cell proliferation and induction of apoptosis were observed in TF-1 $\mathrm{KIT}^{\mathrm{D} 816 \mathrm{~V}}$ cells (Fig. 2b, c). Moreover, the inhibition of the $\mathrm{KIT}^{\mathrm{D} 816 \mathrm{~V}}$-dependent autophagy resulted in a strong reduction of the clonogenic properties of the mutated cell line in semi-solid culture (Fig. 2d, e). We also used AML cell lines that harbored the $K I T^{\mathrm{N} 822 \mathrm{~K}}$ mutation (SKNO-1) or no mutation (U937). The SKNO-1 cell line constitutively expressed a high basal LC3-II level and downregulation of Atg12 and Vps34 by shRNA inhibited cell proliferation and the clonogenic properties (Supplementary Fig. S2A-F). In contrast, in the U937 AML cell line, Atg12 or Vps34 invalidation had no impact on cell proliferation (Supplementary Fig. S2A-D). Altogether, these results demonstrate that KIT mutations induce autophagy that contributes to cell survival and proliferation in AML cells.

\section{Invalidation of Atg12 reduces tumor burden in vivo and prolongs mouse survival}

To assess the role of autophagy in vivo, we used immunocompromised NSG mice engrafted with TF-1 $\mathrm{KIT}^{\mathrm{D} 816 \mathrm{~V}} / \mathrm{sh}$ Atg12 cells by intravenous injection. After establishment of the disease, mice were treated with vehicle or doxycycline to induce Atg12 downregulation and to inhibit autophagy (Fig. 3a). Ten days after doxycycline treatment, the levels of Atg12 and LC3-I/II were strongly downregulated (Fig. 3b-d). The selective knockout of Atg12 was associated with a strong decrease 


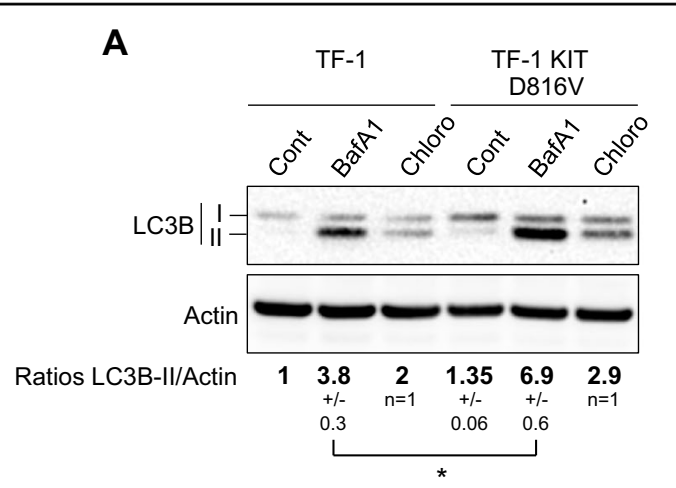

B

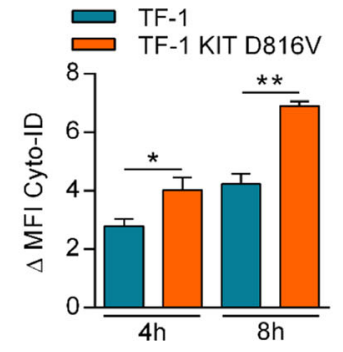

C

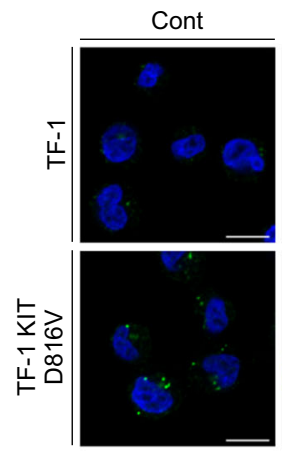

E
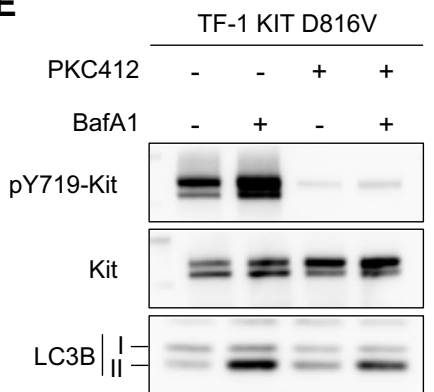

Actin

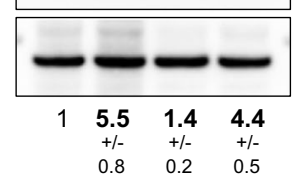

$(n=2)$
BafA1
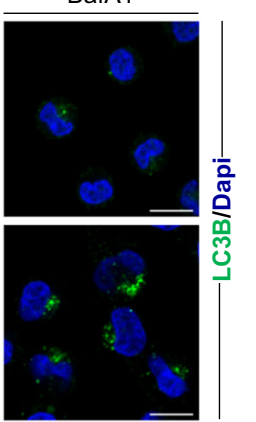

D

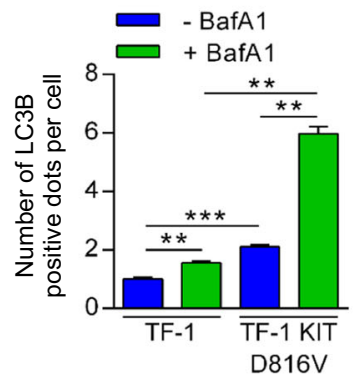

$\mathbf{F}$
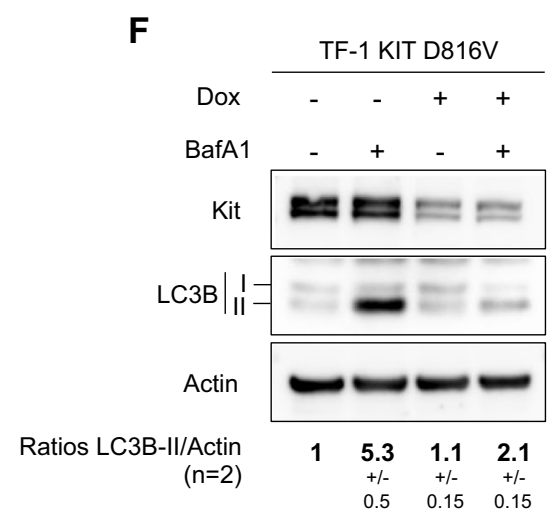

Ratios LC3B-II/Actin

Fig. 1 KIT ${ }^{D 816 V}$ mutation increases autophagic flux in AML cells a-d Oncogenic KIT ${ }^{D 816 V}$ drives autophagy. a TF-1 or TF-1 KIT ${ }^{D 816 V}$ cells were incubated for $4 \mathrm{~h}$ with PBS, Bafilomycin A1 ( $20 \mathrm{nM}, n=3 \pm$ SEM), or chloroquine ( $20 \mu \mathrm{M}, n=2 \pm \mathrm{SD}$ ), and were then analyzed by immunoblotting using the appropriate antibodies. Numbers represent the LC3B-II/actin ratios obtained by densitometric analysis. $\mathbf{b}$ Cells were incubated for 4 or $8 \mathrm{~h}$ with vehicle or $20 \mu \mathrm{M}$ of chloroquine, and then incubated with Cyto-ID before flow cytometry $(n=3 \pm \mathrm{SEM}) . \triangle \mathrm{MFI}=\mathrm{MFI}(\mathrm{chloroquine})-\mathrm{MFI}$ (vehicle). c Cells were treated with vehicle or BafA1 for $2 \mathrm{~h}$ before LC3 staining, fluorescent labeling, and immunofluorescence analysis. $\mathbf{d}$ Quantification of LC3-positive autophagosomes $(n=3 \pm$ SEM). e, $\mathbf{f}$ Pharmacological inhibition or genetic invalidation of a decrease in KIT autophagic flux. e TF-1 KIT ${ }^{\mathrm{D} 816 \mathrm{~V}}$ cells were treated overnight with PKC412 at $1 \mu \mathrm{M} ; 20 \mathrm{nM}$ of BafA1 was added at $2 \mathrm{~h}$ before cell lysis and western blotting. Numbers represent the LC3B-II/actin ratios obtained by densitometric analysis $\left(n=2 \pm\right.$ SD). $\mathbf{f ~ K I T ~}{ }^{\mathrm{D} 16 \mathrm{~V}}$ cells were incubated for 3 days with doxycycline to induce expression of shRNA against KIT. Cells were treated for $2 \mathrm{~h}$ with BafA1 $(20 \mathrm{nM})$ and then analyzed by western blotting. Numbers represent the LC3B-II/actin ratios obtained by densitometric analysis $(n=2 \pm \mathrm{SD})$

of tumor cell burden when compared with vehicle treatment, as indicated by the reduced percentage of human leukemic cells $(\mathrm{hCD} 45+)$ in murine bone marrow and the spleen (Fig. 3e, f). Furthermore, downregulation of
Atg12 significantly prolonged mouse survival $(p=0.0076)$ (Fig. 3g).

To confirm these results in another model, we used murine $\mathrm{Ba} / \mathrm{F} 3$ cells that stably expressed hKIT ${ }^{\mathrm{WT}}$ or 


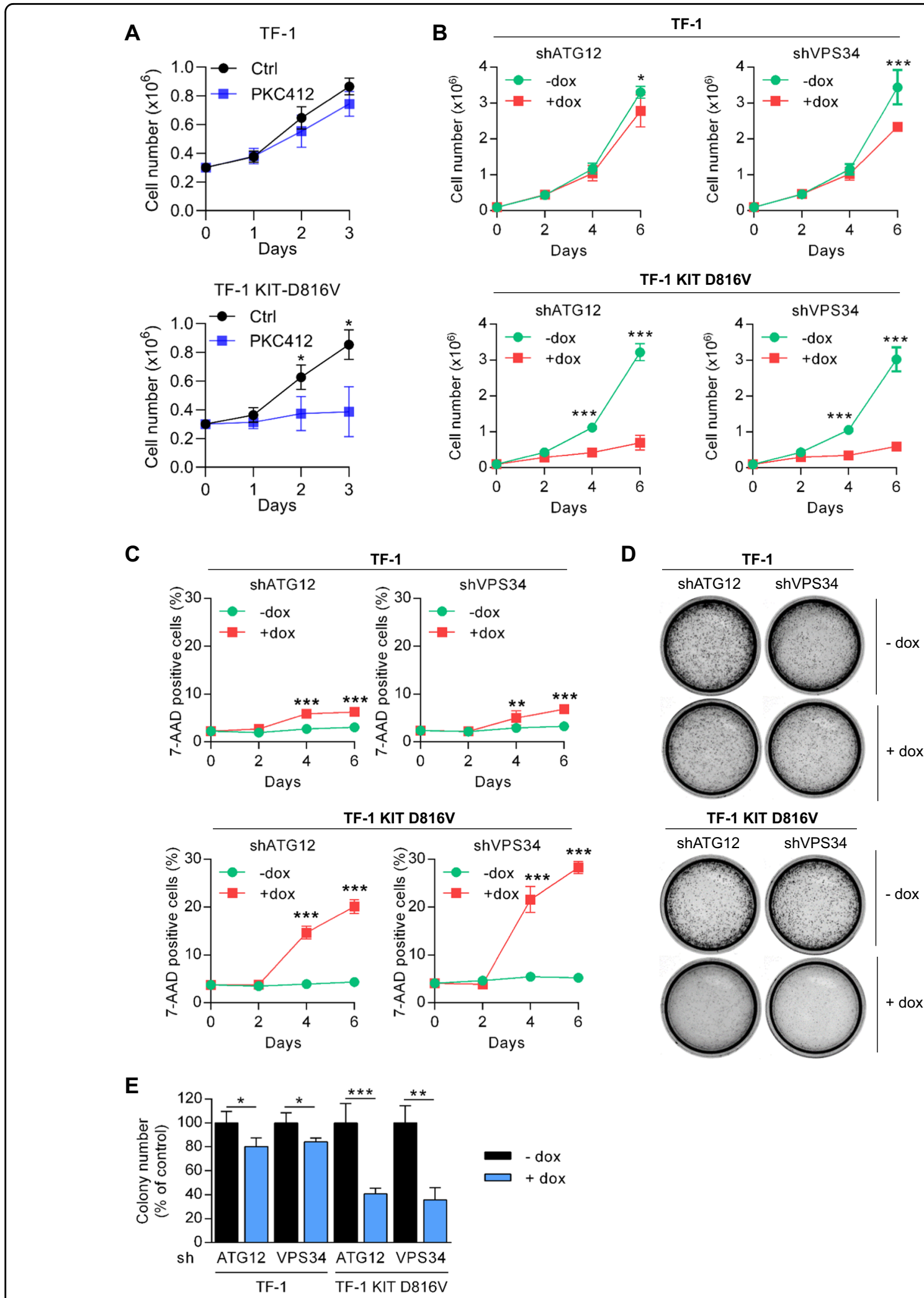

Fig. 2 (See legend on next page.) 
(see figure on previous page)

Fig. 2 KIT-induced autophagy sustains cell proliferation and cell survival. a Impact of pharmacological inhibition of KIT on cell proliferation. TF-1 and TF-1 KIT ${ }^{\text {D816V }}$ cells were treated with PKC412 at $1 \mu \mathrm{M}$ for 3 days and cell proliferation was evaluated by Trypan Blue exclusion counting $(n=3 \pm$ SEM). $\mathbf{b}-\mathbf{e}$ Consequences of invalidation of ATG12 and VPS34, two key autophagic proteins. TF-1 and TF-1 KIT ${ }^{\mathrm{D} 816 \mathrm{~V}}$ cells were transduced with inducible shRNA against ATG12 or VPS34. Target proteins were invalidated after treatment with doxycycline $(1 \mu \mathrm{g} / \mathrm{mL})$. Effects of shRNA on cell proliferation (b), measured by Trypan blue exclusion ( $n=3 \pm$ SEM) and cell death (c) assessed after 7-AAD staining and flow cytometry $(n=3 \pm$ SEM). d, e Colony-forming cell (CFC) assays. $\mathbf{d}$ shRNA-expressing cells were grown in methylcellulose medium in the presence of vehicle or doxycycline $(5 \mu \mathrm{g} / \mathrm{mL})$. e Quantification of colonies ( $n=3 \pm$ SEM)

\section{A}

DO

IV Injection of TF-1 KIT D816V cells in D10

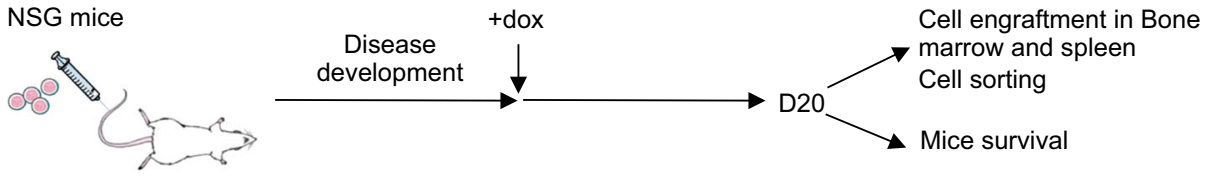

B

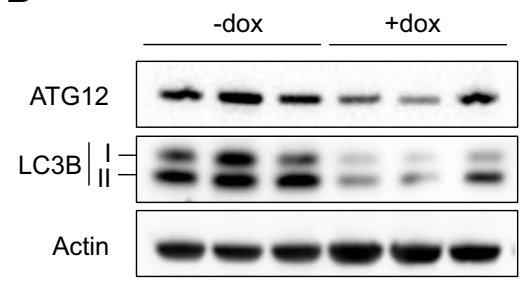

C

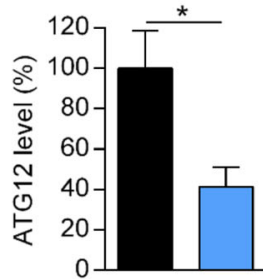

D

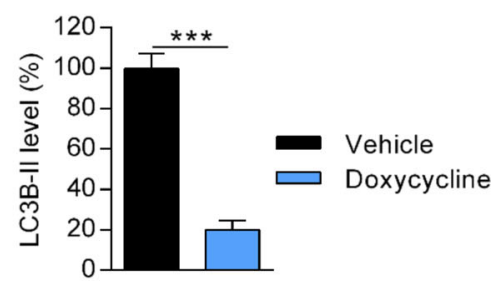

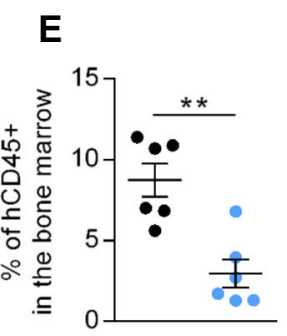
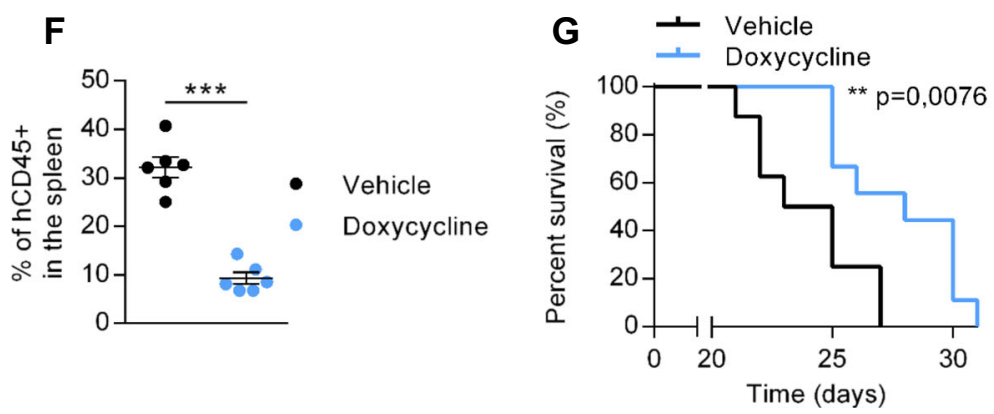

Fig. 3 Targeting autophagy decreased leukemic development of human $\mathrm{KIT}^{\mathrm{D} 816 \mathrm{~V}}$ cells when xenografted into NSG mice. A total of $2 \times 10^{6}$ of TF-1 KITD816V cells expressing inducible shRNA against ATG12 were injected into the vein tails of NSG mice and doxycycline was added on day 10. On day 20, six mice per group were killed: the human cells were studied by flow cytometry and sorted. Overall mouse survival was also monitored. a Experimental procedure. $\mathbf{b}$ After killing the mice, human leukemic cells were isolated with hCD45 magnetic beads and analyzed by western blotting. c, $\mathbf{d}$ Quantification of protein level of ATG12 and LC3-II. Numbers represent the LC3B-II/actin ratios obtained by densitometric analysis ( $n=3 \pm$ SEM). e, $\mathbf{f}$ Percentage of human hCD45+ cells in the bone marrow (e) and spleen (f) of mice evaluated by flow cytometry $(n=6 \pm$ SEM). $\mathbf{g}$ Overall survival of mice (vehicle $n=8$, doxycycline $n=9$ )

$\mathrm{hKIT}^{\mathrm{D} 816 \mathrm{~V}}$. A higher autophagic flux was observed in cells harboring hKIT ${ }^{\mathrm{D} 816 \mathrm{~V}}$ mutation compared with hKIT ${ }^{\mathrm{WT}}$ cells (Supplementary Fig. S3A). Using inducible shRNA against Atg12, which reduced LC3B-II level (Supplementary Fig. S3B), we showed that inhibition of autophagy significantly decreased cell proliferation in vitro and leukemic progression in vivo of $\mathrm{Ba} / \mathrm{F} 3-\mathrm{KIT}^{\mathrm{D} 816 \mathrm{~V}}$ cells (Supplementary Fig. S3C-D). Thus, these results demonstrate that $\mathrm{KIT}^{\mathrm{D} 816 \mathrm{~V}}$-induced autophagy is necessary to drive leukemic progression in vivo, and that autophagy inhibition could represent a valuable therapeutic target in AML with KIT mutations. 


\section{KIT mutant induces autophagy through STAT3 activation in AML cells}

The oncogenic properties of $\mathrm{KIT}^{\mathrm{D} 816 \mathrm{~V}}$ are mediated by constitutive activation of STAT3/5, mitogen-activated protein kinase (MAPK), and PI3K/AKT pathways. As these signaling pathways modulate autophagy, we sought to determine which downstream target of $\mathrm{KIT}^{\mathrm{D} 816 \mathrm{~V}}$ drives autophagy in this model. We first compared cell signaling in TF- 1 cells and in TF- $1 \mathrm{KIT}^{\mathrm{D} 816 \mathrm{~V}}$, and observed that, as expected, TF-1 KIT $^{\mathrm{D} 816 \mathrm{~V}}$ displayed constitutive phosphorylation of STAT3, ERK, and AKT compared with TF1 cells (Fig. 4a). Interestingly, the wild-type KIT receptor, once activated by its ligand in both TF-1 and OCI-AML3 AML cells, induced, as observed in constitutively activated KIT $^{\text {D816V }}$ mutant cells, (Supplementary Fig. S4A-E) autophagy and activation of STAT3, ERK, and AKT pathways (Supplementary Fig. S4F). We then assessed the impact of pharmacological inhibitors in these pathways on autophagic flux in TF-1 KIT ${ }^{\mathrm{D} 816 \mathrm{~V}}$ cells and in cells expressing the wild-type KIT receptor upon its activation by the stem cell factor (SCF). Inhibition of ERK by PD0325901 had no impact on autophagic flux, whereas the AKT inhibitor increased it, likely through mammalian target of rapamycin (mTOR) inhibition (as expected; Fig. 4 b, c and Supplementary Fig. S4G).

In contrast, Stattic, a small-molecule inhibitor of STAT3, significantly reduced the accumulation of LC3-II upon Bafilomycin A treatment in TF-1 KIT ${ }^{\mathrm{D} 816 \mathrm{~V}}$ cells (Fig. 4b) but also in TF-1 or OCI-AML3 when stimulated with SCF (Supplementary Fig. S4GH), indicating a reduced autophagic flux upon STAT3 inhibition. Similar results were obtained using cyto-ID staining and immunofluorescence analysis (Fig. 4c-f and Supplementary Fig. S4G, IJ). These results were recapitulated with another STAT3 inhibitor, WP1066 (Fig. 4d-f and Supplementary Fig. S4H-J). We then transduced TF-1 KIT ${ }^{\mathrm{D} 816 \mathrm{~V}}$ with lentiviral vectors encoding two inducible shRNAs against STAT3. In these cells incubated with Bafilomycin A, the selective knockdown of STAT3 decreased the conversion from LC3-I to LC3-II and the number of LC3-positive structures seen in immunofluorescence microscopy (Fig. $4 \mathrm{~g}-\mathrm{i})$. Altogether, these data demonstrate that activated KIT, either constitutively or upon SCF stimulation, induces autophagy through STAT3 activation in AML cells.

To decipher the link between STAT3 activation and autophagy induction, we performed a transcriptomic analysis on TF-1 cells expressing either the wild type or the mutated form of KIT (Supplementary Fig. S5A). Consistently with the activation of STAT3 (Supplementary Fig. S5B), STAT3-regulated genes were found overexpressed in $\mathrm{KIT}^{\mathrm{D} 816 \mathrm{~V}}$-expressing cells compared with wild-type cells (Supplementary Fig. S5A,C). Indeed, the expression of a previously identified STAT3 gene signature ${ }^{25}$ (DAUER_STAT3_TARGETS_UP) was significantly enriched as indicated by Gene Set Enrichment Analysis (GSEA) (Supplementary Fig. S5C). However, autophagy-related genes controlled by STAT3 were not differentially expressed between the two cell lines. Accordingly, the expression of two identified autophagy signatures (GO_REGULATION_OF_AUTOPHAGY; KEGG_REGULATION_OF_AUTOPHAGY) are not enriched in $\mathrm{KIT}^{\mathrm{D} 816 \mathrm{~V}}$ cells and are rather enhanced in wild-type cells (Supplementary Fig. S5D). Together, these results are not in favor of a transcriptional regulation of autophagy by STAT3 in this model.

\section{In vivo targeting of STAT3 inhibits autophagy and reduces tumor cell burden in $\mathrm{KIT}^{\mathrm{D} 816 \mathrm{~V}}$ AML cells}

To inhibit STAT3 activity and to perform a preclinical proof-of-concept that STAT3 is an effective molecular target for AML cells expressing mutated KIT, we used the Stattic inhibitor directly injected into the tumor as previously described in vivo ${ }^{26,27}$. Therefore, we established a xenograft model using NOD/SCID mice that were subcutaneously injected with TF-1 KIT ${ }^{\mathrm{D} 816 \mathrm{~V}}$ cells. After tumor establishment, the mice were treated with daily intra-tumoral injections of Stattic ( 2 or $4 \mathrm{mg} / \mathrm{kg} /$ day) or vehicle. Stattic treatment reduced STAT3 phosphorylation, strongly downregulated LC3-II expression, especially at the higher dosage, and significantly inhibited tumor growth. This indicates that pharmacological inhibition of STAT3 inhibits autophagy in vivo resulting in significant anti-leukemic activity (Fig. 5a-f).

\section{Discussion}

Similar to other oncogenes, including $B R A F^{V 600 E}$ or $K R A S^{G 12 D}$ in solid tumors ${ }^{8,9,28,29}$ or $B C R-A B L$ in chronic myelocytic leukemia ${ }^{11}$ and FLT3-ITD in $\mathrm{AML}^{16}$, our study shows that activating mutations of the tyrosinekinase receptor KIT triggers autophagy and supports cell proliferation and survival in AML cells. Very recent insights into the AML cell metabolism have revealed that several metabolic pathways (e.g., glucose, glutamine, or fatty acid) regulated by autophagy, are crucial for AML cell growth and survival. Thus, RTK mutations, including KIT and FLT3-ITD, could activate autophagy to maintain a sufficient pool of energy substrates to sustain tumor growth and chemoresistance ${ }^{30}$. Indeed, it has been recently shown that glutamine metabolism is required for energy production through the tricarboxylic acid cycle (TCA) cycle and for redox homeostasis in AML cells ${ }^{31,32}$. Similarly, fatty acid uptake and high oxidative metabolism appear to be key players in the chemoresistance of $\mathrm{AML}^{33-35}$. Whether KIT-driven AML cells are preferentially dependent on specific metabolic substrates remains to be determined.

As far as hematological malignancies are concerned, autophagy has been mainly described as a mechanism of 


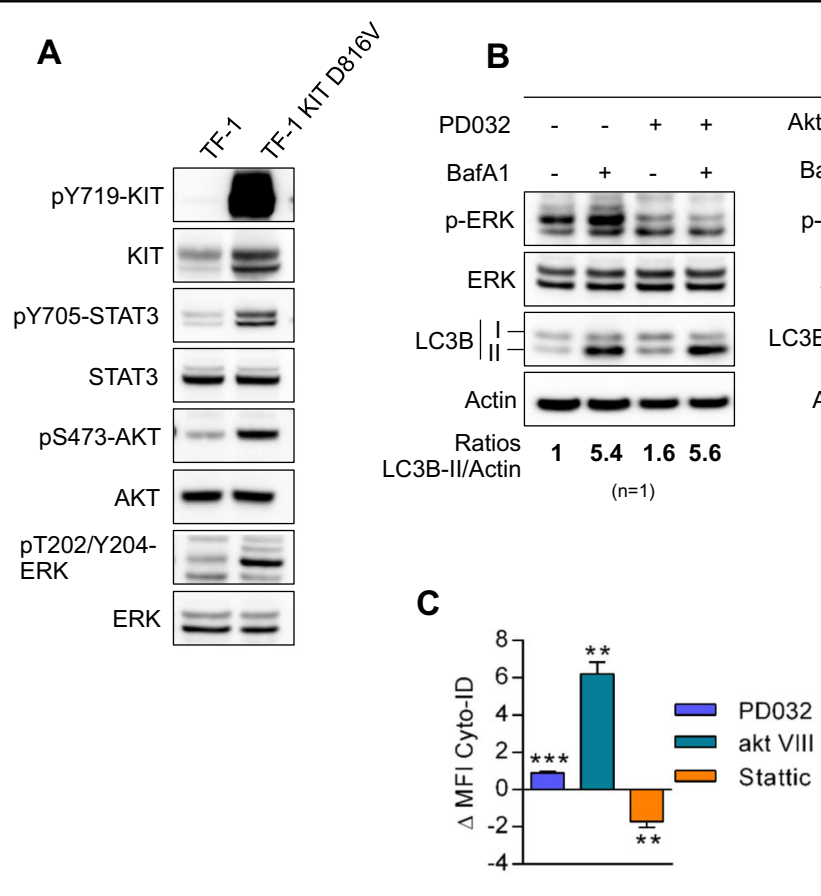

TF-1 KIT D816V

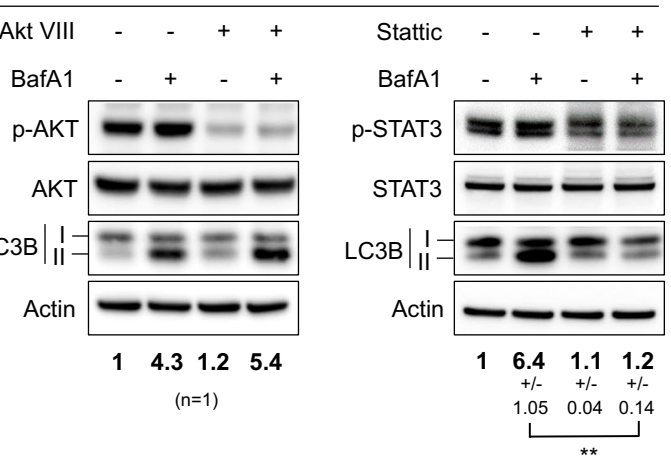

D

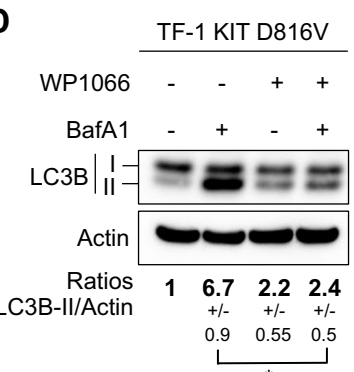

E

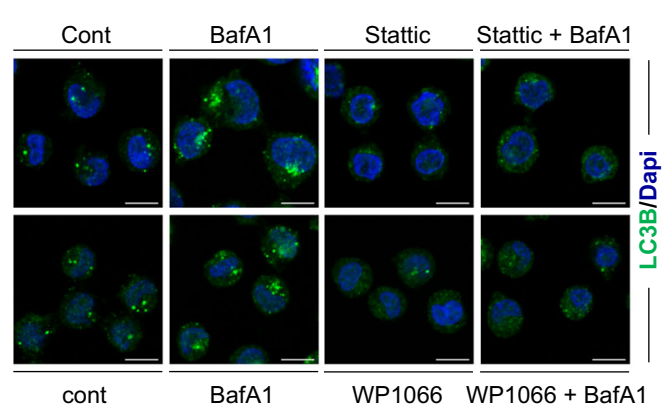

F

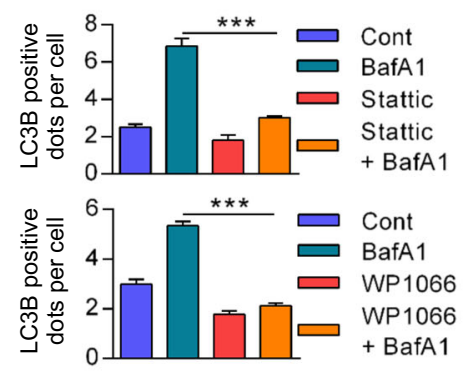

G

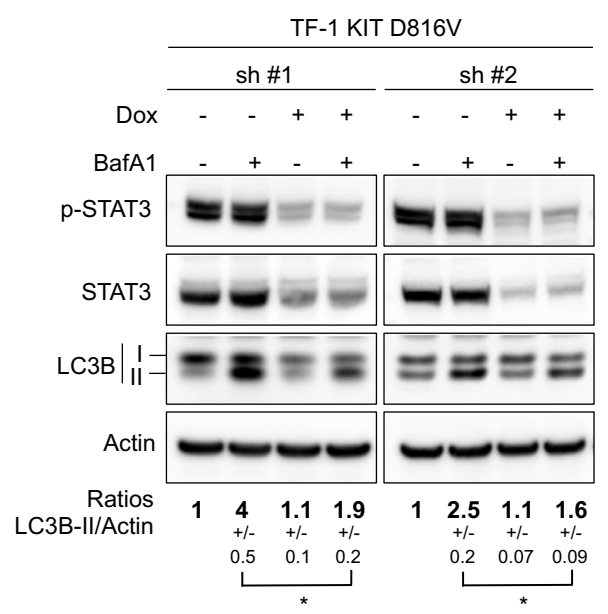

H

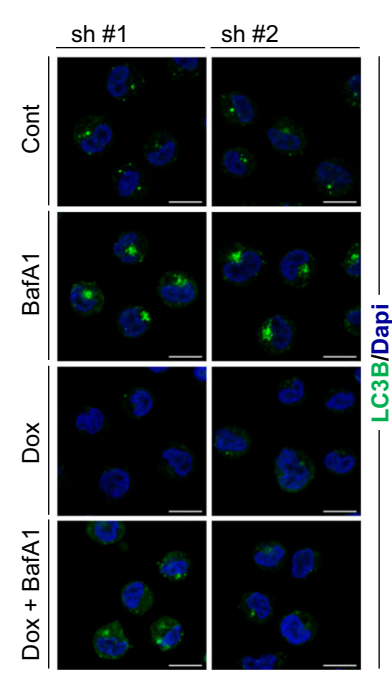

I
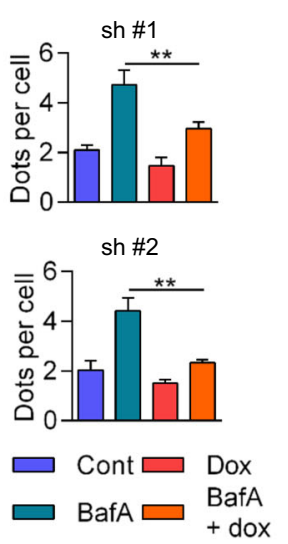

Fig. 4 (See legend on next page.) 
(see figure on previous page)

Fig. 4 STAT3 drives autophagy in $\mathrm{KIT}^{\mathrm{D} 816 \mathrm{~V}}$ cells. a Comparison of cell signaling in TF-1 and TF-1 $\mathrm{KIT}^{\mathrm{D} 816 \mathrm{~V}}$ cells. $\mathbf{b}$ Identification of the signaling pathway involved in $\mathrm{KIT}^{\mathrm{D} 816 \mathrm{~V}}$-induced autophagy. TF-1 KIT ${ }^{\mathrm{D} 816 \mathrm{~V}}$ cells were treated for $2 \mathrm{~h}$ with PBS and BafA 1 at $20 \mathrm{nM}$ alone or in association with the indicated inhibitors. PD0325901 was used at $100 \mathrm{nM}(n=1)$, Akt inhibitor-VIII at $1 \mu \mathrm{M}(n=1)$, and Stattic at $10 \mu \mathrm{M}(n=3 \pm$ SEM). The cells were then lysed and analyzed by immunoblotting. Numbers represent the LC3B-II/actin ratios obtained by densitometric analysis. c Assessment of autophagic flux by flow cytometry after Cyto-ID labeling. Cells were incubated with $10 \mu \mathrm{M}$ of chloroquine alone or in combination with PD03259, Akt inhibitorVIII, or Stattic, as previously described. $\triangle \mathrm{MFI}=\mathrm{MFI}$ (chloroquine + inhibitor) - MFI(chloroquine) $(n=3 \pm$ SEM). d STAT3 inhibitor WP1066 was added at $15 \mu \mathrm{M}$ alone or in combination with $20 \mathrm{nM}$ of BafA1 at $2 \mathrm{~h}$ before western blotting. Numbers represent the LC3B-II/actin ratios obtained by densitometric analysis $(n=3 \pm$ SEM). e Cells were treated for $2 \mathrm{~h}$ with vehicle, BafA1, Stattic, or WP1066 alone or in combination with BafA 1 before LC3 staining, fluorescent labeling, and immunofluorescence analysis. $\mathbf{f}$ Quantification of LC3-positive autophagosomes $(n=3 \pm$ SEM). $\mathbf{g}-\mathbf{i}$ Genetic invalidation of STAT3. TF-1 KIT ${ }^{\text {D816V }}$ cells were transduced with two different inducible shRNAs against STAT3. Doxycycline $(1 \mu \mathrm{g} / \mathrm{mL}) \mathrm{was}$ added 3 days before the experiment. Autophagic flux was assessed by western blotting $\mathbf{g}$ with numbers representing the LC3B-II/actin ratios obtained by densitometric analysis $(n=3 \pm$ SEM) and by immunofluorescence (h) on cells treated \pm dox and $\pm 20 \mathrm{nM}$ BafA1. i Quantification of LC3-positive autophagosomes $(n=3 \pm$ SEM)

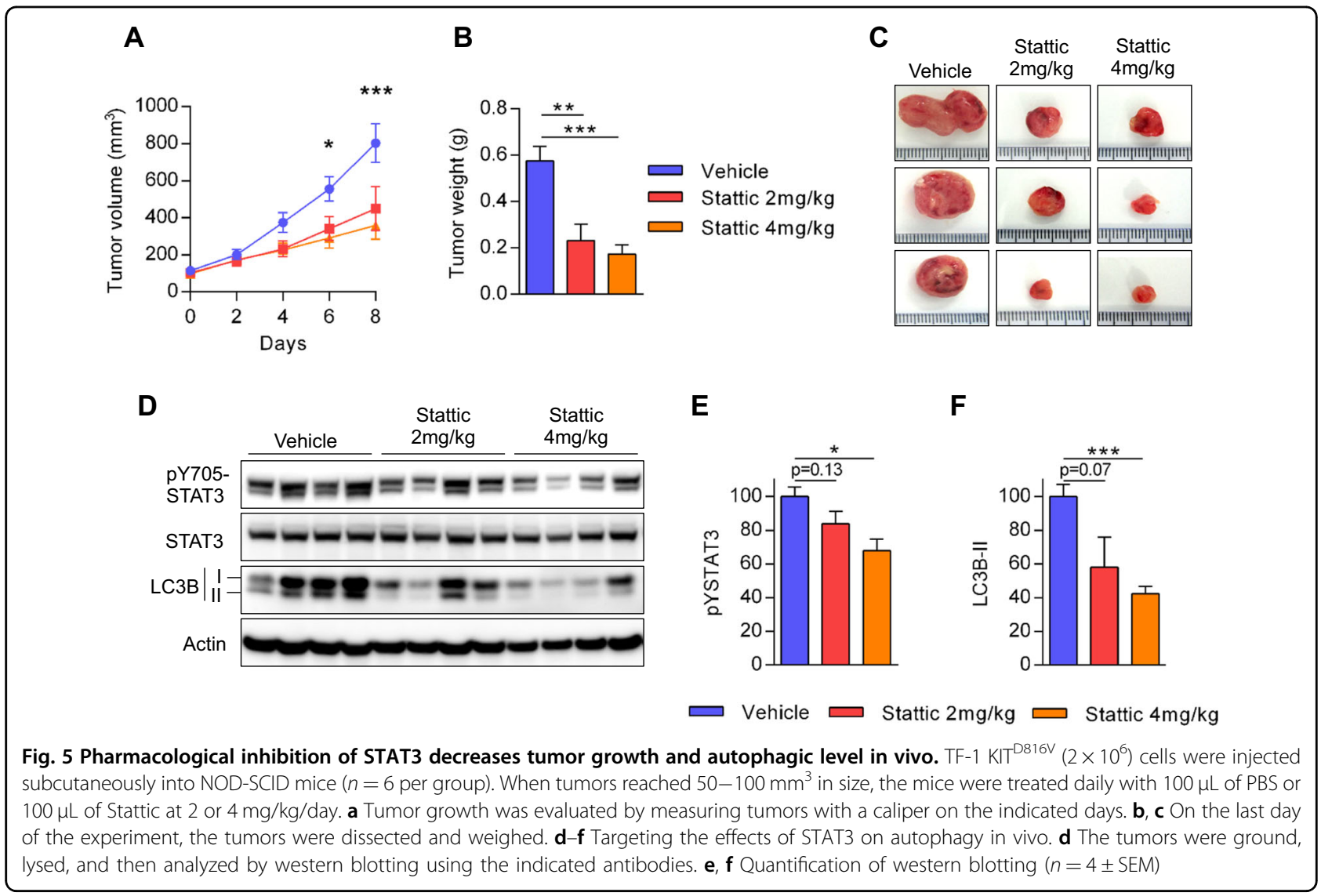

resistance to cytotoxic agents or tyrosine-kinase inhibitors, including cytarabine or imatinib ${ }^{10,14}$. It has been shown that following invalidation of the key autophagic genes, ATG7 or ATG5, AML cells were sensitized to the anti-leukemic activity of cytarabine in vivo and prolonged mouse survival. Thus, it is reasonably conceivable that the introduction of new potent autophagic inhibitors into the armamentarium of anti-AML therapies could be of benefit in KIT-mutated $\mathrm{AML}^{36-38}$. It is also noteworthy that, in sharp contrast to imatinib or crizotinib that induce cytoprotective autophagy, midostaurin (PKC412), a pantyrosine-kinase inhibitor that potently targets KIT, decreased autophagy flux in KIT-mutated AML cells ${ }^{10,39}$. Midostaurin, which is active in advanced systemic mastocytosis with $K I T^{D 816 V}$ mutations, was recently found to significantly increase overall survival in a phase-3 clinical trial on AML patients with FLT3 mutations ${ }^{40}$. Our results suggest that CBF-AML with a KIT mutation should also benefit from midostaurin treatment through inhibition of cell signaling without activating cytoprotective autophagy. 
STAT3 has been involved as a positive or negative regulator of autophagy, depending on the cellular context and its subcellular localization patterns ${ }^{41}$. On the one hand, it has been shown that cytoplasmic STAT3 repressed autophagy in osteosarcoma U2OS cells by inhibiting PKR activity ${ }^{42}$. In these cells, pharmacological inhibitors of STAT3, i.e., WP1066 or Stattic, strongly increased autophagy flux. On the other hand, some studies suggest that following phosphorylation on serine 727 by ERK or JNK kinases, STAT3 is translocated to the mitochondria where it supports cellular respiration and autophagy ${ }^{43-47}$.

In addition, STAT3 is a transcriptional enhancer of several autophagy-related genes in the nucleus and this activity contributes to a range of anti- or pro-autophagic functions of STAT3 in autophagy ${ }^{41}$. For example, it has been demonstrated that the expression of anti-apoptotic genes, $B C L 2$ and $M C L 1$, are increased by STAT3 ${ }^{48,49}$, whereas $B E C N 1$ is downregulated ${ }^{50}$, resulting in antiautophagic functions of STAT3. In contrast, STAT3 can enhance HIF1A or BNIP3 expression, which is associated with an increase in autophagy ${ }^{51,52}$. More recently, interleukin (IL)-6-induced autophagy has been shown in hypoxic glioblastoma cells via the p-STAT3-MIR155-3pCREBRF pathway ${ }^{53}$. KIT-activating mutations are associated with both Y705 and S727 phosphorylation of STAT3, leading to nuclear translocation and transactivation of target genes ${ }^{54,55}$. Thus, the pro-autophagic functions of STAT3 in our model could have been the result of this enhanced nuclear activity. However, our transcriptomic analysis (Fig. 5b-d) performed on cells expressing the mutated KIT is more in favor of a transcription-independent role of STAT3 on autophagy modulation. A function for cytoplasmic localized STAT3 is then more likely to be implicated and further studies are needed to fully assess the role of STAT3 in autophagy downstream of activated KIT.

Therapeutic approaches that exploit autophagy properties or target STAT3 represent a new field of investigation in hematological cancers. Classic pharmacological inhibitors of autophagy, including hydroxychloroquine, are being tested in combination with chemotherapy to treat AML, whereas new more specific compounds are currently being evaluated in early clinical trials ${ }^{56}$. Our study suggests that certain molecular subtypes of AML could be more susceptible to responding to inhibition of autophagy, thus indicating that results from clinical trials that are assessing autophagy inhibitors will need to take the genetic heterogeneity of AML into account.

\section{Materials and methods \\ Cell lines}

TF-1 and TF-1 KIT ${ }^{\mathrm{D} 816 \mathrm{~V}}$ cell lines were a kind gift from Patrice Dubreuil laboratory (Marseille, France). TF-1 was grown in RPMI containing 10\% fetal calf serum (FCS) supplemented with $2 \mathrm{ng} / \mathrm{ml}$ granulocyte macrophage colony-stimulating factor (GM-CSF) (Miltenyi Biotech, France). At $4 \mathrm{~h}$ before the experiments, cells were washed and maintained in GM-CSF-free medium. TF-1 KIT ${ }^{\mathrm{D} 816 \mathrm{~V}}$ and OCI-AML3 cells were cultured in RPMI 1640 medium with Glutamax (Gibco, Life Technologies) supplemented with fetal bovine serum (Sigma) and 100 units $/ \mathrm{mL}$ of penicillin and streptomycin (Invitrogen). Human myeloid-leukemia cell lines SKNO-1 and U937 were purchased from the Leibniz Institute DSMZ (the German Collection of Microorganisms and Cell Cultures, Leibniz, Germany) and cultured in RPMI containing 10\% FCS and penicillin/streptomycin.

\section{Antibodies and reagents}

An antiphospho-Kit (Y719), anti-Kit (D13A2), antiLC3-B (for immunoblotting), antiphosphoStat-3 (Y705), antiphospho-Akt (S473), anti-Akt, antiphospho-p44/42 MAPK Erk1/2 (T202/Y204), and anti-p44/42 MAPK Erk1/2 were obtained from Cell Signaling Technology (Beverly, MA, USA); anti-STAT3 was obtained from Santa Cruz Biotechnology (CA, USA). For the immunofluorescence analysis, mouse monoclonal anti-Lc3-B (Nanotools, Germany) was used.

Secondary antibodies labeled with horseradish peroxidase were purchased from Promega. Stattic, PKC412, and WP1066 were purchased from Selleck Chemicals (Houston, TX, USA), Bafilomycin A1 was from Invivogen (Toulouse, France), and chloroquine and doxycycline hyclate were from Sigma (St. Louis, MO, USA). SCF was purchased from Miltenyi Biotech and used at $100 \mathrm{ng} / \mathrm{ml}$ concentration. The antibodies anti-hCD45 (YB5.B8, from BD Pharmingen), Annexin-V, 7-amino-actinomycin D (7AAD) (Sigma), and a BD Via-Probe ${ }^{\mathrm{TM}}$ (BD Pharmingen) were used in the flow cytometry studies.

\section{Western blotting analysis}

Proteins were resolved using 4-12\% n-polyacrylamide electrophoresis Bis-Tris gels (Life Technology, Carlsbad, CA, USA) and electro-transferred to nitrocellulose membranes. After blocking in phosphate-buffered saline (PBS) $-0.1 \%$ Tween-20 and bovine serum albumin (BSA), the membranes were immunostained with appropriate antibodies and horseradish peroxidase-conjugated secondary antibodies. Immunoreactive bands were visualized by enhanced chemiluminescence (PI32209; Thermo Fisher Scientific) with a Syngene camera. Densitometric analyses of immunoblots were performed using GeneTools software.

\section{Cell-death assay}

To assess cell death, $5 \times 10^{5}$ cells were washed with PBS and then resuspended in $200 \mu \mathrm{L}$ of PBS. 7-AAD $(2 \mu \mathrm{L})$ 
was added at room temperature and in darkness. All samples were analyzed by a fluorescence-activated cell sorter (FACS: Calibur-Flow Cytometer, BD Pharmingen, San Diego, USA).

\section{Lentiviral infection of TF-1 cells}

Lentiviral particles were generated by transient calciumphosphate transfection in 293T cells. Briefly, 293T was placed in a $10 \mathrm{~cm}$ dish and transfected with $62.5 \mu \mathrm{L}$ of $\mathrm{CaCl}_{2}(2 \mathrm{M}), 500 \mu \mathrm{L}$ of $\mathrm{HeBS} 2 \times, 418 \mu \mathrm{l}$ of $\mathrm{H}_{2} \mathrm{O}, 3.5 \mu \mathrm{g}$ of pVSV-G (env), $6.5 \mu \mathrm{g}$ of p8.1 (tat, pol, rev, gag), and $10 \mu \mathrm{g}$ of inducible TRIPZ human shRNA (GE Healthcare, CO, USA) against ATG12 (RHS4696-20075354), VPS34 (V3THS_372038), STAT3 (V2THS_262105 or V3THS_376016), or KIT (V2THS_76974). At $48 \mathrm{~h}$ after transfection of 293T, $2 \mathrm{~mL}$ of supernatant containing the lentiviral particles was incubated with $\sim 2 \times 10^{6}$ cells in six-well plates. Polybrene was added to make a final concentration of $8 \mu \mathrm{g} / \mathrm{mL}$. Spinoculation was performed by centrifuging cells for $45 \mathrm{~min}$ at $800 \times g$.

On the day after transduction, the medium containing the virus was removed and changed for a virus-free medium. After an additional $24 \mathrm{~h}$, the cells were selected using $1 \mu \mathrm{g} / \mathrm{mL}$ of puromycin. When puromycin-resistant cells appeared, the cells expressing a high level of shRNA (Red Fluorescent Protein-positive cells) were sorted by flow cytometry after $24 \mathrm{~h}$ of treatment with $1 \mu \mathrm{g} / \mathrm{mL}$ of doxycycline. All shRNA experiments were performed on bulk cells treated or not with $1 \mu \mathrm{g} / \mathrm{mL}$ of doxycycline for shRNA induction.

\section{Immunofluorescence microscopy}

After the above treatments, the cells were placed onto poly-L-lysine-coated slides. Cells were then fixed in $4 \%$ formaldehyde for $8 \mathrm{~min}$, and then for $5 \mathrm{~min}$ at $4{ }^{\circ} \mathrm{C}$ with $100 \%$ methanol. The cells were then permeabilized for 5 min with PBS containing $0.5 \%$ Triton X-100 and saturated for $30 \mathrm{~min}$ in PBS-5\% BSA. The LC3B antibody $(2 \mu \mathrm{g} / \mathrm{mL})$, in PBS-5\% BSA, was added $1 \mathrm{~h}$ at room temperature, followed by washing and $30 \mathrm{~min}$ of incubation in secondary antibody conjugated with Alexa-488, Alexa555, or Alexa-647 (Invitrogen). Slides were mounted in Prolong Gold Antifade Mountant with 4',6-diamidino-2phenylindole (DAPI) (Invitrogen) and visualized on a Zeiss LSM710 or Zeiss LSM780 microscope using ZEN 2012 software. For quantification, fields were chosen arbitrarily based on DAPI staining and the number of LC3B dots per cell of at least 50 cells was determined using Image $\mathrm{J}$ software.

\section{Cyto-ID ${ }^{\circledR}$}

Autophagic flux was assessed using the Cyto-ID ${ }^{\circledR}$-based procedure according to the manufacturer's instructions (Enzo Life Sciences, Switzerland). The fluorescence of the
Cyto-ID ${ }^{\circledR}$ dye incorporated into the different AML cells were analyzed by a FACS: Calibur-Flow Cytometer (BD Pharmingen, San Diego, USA; LSR Fortessa, BD Pharmigen).

\section{RNA microarray and bioinformatics analyses}

Total RNA were extracted form TF-1 cells expressing either the wild-type KIT or the mutant $\mathrm{KIT}^{\mathrm{D} 816 \mathrm{~V}}$ in independent triplicate using the Qiagen kit according to manufacturer's instructions. RNA purity was monitored with NanoDrop 1ND-1000 spectrophotometer and RNA quality was assessed through Agilent 2100 Bionalyzer with RNA 6000 Nano assay kit. No RNA degradation or contamination were detected (RIN >9). One hundred nanograms of total RNA were analyzed on Affymetrix GeneChip $\odot$ Human Gene 2.0 ST Array using the Affymetrix GeneChip@ $\odot$ WT Plus Reagent Kit according to the manufacturer's instructions (Manual Target Preparation for GeneChip ${ }^{\circledR}$ Whole Transcript (WT) Expression Arrays P/N 703174 Rev. 2). Arrays were washed and scanned, and the raw files generated by the scanner was transferred into Partek@ Genomics Suite for preprocessing (with default partek option). Quality control (boxplot, clustering and PCA) and differential expression analysis (with eBayes function, LIMMA package) were performed in R/CRAN environment. Mapping between transcript clusters and genes were done using annotation provided by Affymetrix (HuGene-2_0-st-v1.na34.hg19.transcript. csv) and the R/Bioconductor package hugene2.0sttranscriptcluster.db. $p$-values generated by the eBayes function were adjusted to control false discovery using the Benjamini and Hochberg's procedure [RMA] Irizarry et al., Biostatistics, 2003; [Oligo package] Carvalho and Irizarry, Bioinformatics, 2010; [LIMMA reference] Ritchie et al., Nucleic Acids Research, 2015; hugene20sttranscriptcluster.db:MacDonald JW 2017, Affymetrix hugene20 annotation data (chip hugene20sttranscriptcluster); [FDR]: Benjamini et al., Journal of the Royal Statistical Society, 1995.

GGSEA analysis were performed using GSEA v3.0 tool developed by the Broad Institute.

Transcriptomic data have been deposited at the Gene Expression Omnibus database (GSE130625).

\section{Tumor xenografts in NOD/SCID IL-2 receptor g-chain-null mice}

Xenograft tumors in NOD/SCID IL-2 receptor g-chainnull mice (NSG) mice of $6-8$ years old were generated by injecting $2 \times 10^{6}$ TF-1 $\mathrm{KIT}^{\mathrm{D} 816 \mathrm{~V}}$ into the tail vein. Ten days after injection, mice were randomly treated either by adding doxycycline $(200 \mu \mathrm{g} / \mathrm{ml})$ and sucrose $(10 \mu \mathrm{g} / \mathrm{ml})$ to the drinking water or by adding sucrose only. Without blinding, human-cell engraftment (hCD45+ cells) into the bone marrow or spleen was evaluated by flow cytometry 
(BD FACS Calibur). Human cells were also isolated by magnetic sorting with hCD45 beads (Miltenyi Biotech, France) and analyzed by western blotting using the appropriate antibodies. Xenograft tumors in NOD/SCID mice were generated by subcutaneously injecting $2 \times 10^{6}$ cells in $100 \mu \mathrm{L}$ of PBS cells into both flanks. Once the tumors had reached $50-100 \mathrm{~mm}^{3}$ in size, without blinding the animals were given $2 \mathrm{mg} / \mathrm{mL}$ or $4 \mathrm{mg} / \mathrm{mL}$ of Stattic or vehicle (PBS). Tumor dimensions were measured with a caliper on the every 2 or 3 days and volume $(v)$ was calculated using the formula: $v=A^{*} B^{2} / 2$, where $A$ is the larger diameter and $B$ is the smaller diameter. All experiments were conducted in accordance with the guidelines of the Association for Assessment and Accreditation of Laboratory Animal Care International.

\section{Statistical analyses}

Data from at least three independent experiments are reported as their means \pm SEM. Statistical analyses were assessed using unpaired two-tailed Student's $t$-test using Prism 5 software (GraphPad Software, Inc., La Jolla, CA, USA). A value of $p<0.05$ was regarded as being significant: *, ***, and ${ }^{* * * *}$ correspond to $p<0.05, p<0.01$, and $p<0.001$, respectively. The Kaplan-Meier method was used to estimate leukemia-free survival in xenografted mice. Log-rank $p$-values were used to compare leukemiafree survival among the subgroups.

\section{Acknowledgements}

We thank all members of the GAEL (Gaël Adolescent Espoir Leucémie) association. We acknowledge Valérie Duplan-Eche, Fatima L'Faqihi-Olive, and Manon Farcé for their technical assistance in the flow-cytometry core facilities at INSERM UMR1043 and INSERM UMR1037. We are grateful to Laetitia Ligat for technical assistance in the imagery facility at INSERM UMR1037. This work was supported by Ligue nationale contre le Cancer and Clément Larrue is a recipient of the Ligue Nationale contre le Cancer. We thank Patrice Dubreuil (CRCM, Marseille, France) for providing the specific leukemic cell lines: TF-1 and TF-1 KIT D816V . This work received a grant from the French government under the "Investissement d'avenir" program (ANR-11-PHUC-001), the Institut National du Cancer (INCA-PLBIO 2012-105), and the InnaBioSanté foundation (RESISTAML project).

\section{Author details}

${ }^{1}$ Cancer Research Center of Toulouse (CRCT), UMR1037 INSERM, ERL5294 CNRS, Equipe Labellisée LIGUE, Toulouse, France. 'University of Toulouse, Toulouse, France. ${ }^{3}$ Proteome and Genome Research Unit, Department of Oncology, Luxembourg Institute of Health, Strassen, Luxembourg. ${ }^{4}$ Service d'Hématologie, Centre Hospitalier Universitaire de Toulouse, Institut Universitaire du Cancer de Toulouse Oncopole, Toulouse, France

\section{Author contributions}

Conception and design: C.L., C.R. Development of methodology: C.L., E.S., J.E.S., C.J., C.R. Acquisition of data (provided animals, acquired and managed AML samples, provided facilities, etc.): C.L., Q.H., E.S., J.E.S., T.K., C.J., C.R. Analysis and interpretation of data: C.L., C.J., C.R. Writing, review, and/or revision of the manuscript: C.L., J.E.S., C.J., C.R. Study supervision: C.R. Revision supervision: C. J.

\section{Conflict of interest}

The authors declare that they have no conflict of interest.

\section{Publisher's note}

Springer Nature remains neutral with regard to jurisdictional claims in published maps and institutional affiliations.

Supplementary Information accompanies this paper at (https://doi.org/ 10.1038/s41389-019-0148-9).

Received: 12 May 2017 Revised: 10 April 2019 Accepted: 31 May 2019 Published online: 16 July 2019

\section{References}

1. Boya, P., Reggiori, F. \& Codogno, P. Emerging regulation and functions of autophagy. Nat. Cell Biol. 15, 713-720 (2013).

2. Choi, A. M. K., Ryter, S. W. \& Levine, B. Autophagy in human health and disease. N. Engl. J. Med. 368, 651-662 (2013).

3. Lorin, S., Hamaï, A., Mehrpour, M. \& Codogno, P. Autophagy regulation and its role in cancer. Semin. Cancer Biol. 23, 361-379 (2013).

4. Joffre, C., Djavaheri-Mergny, M., Pattingre, S. \& Giuriato, S. [The yin and the yang of autophagy in cancer cells]. Med. Sci. Ms. 33, 328-334 (2017).

5. Yue, Z., Jin, S., Yang, C., Levine, A. J. \& Heintz, N. Beclin 1, an autophagy gene essential for early embryonic development, is a haploinsufficient tumor suppressor. Proc. Natl Acad. Sci. USA 100, 15077-15082 (2003).

6. $\mathrm{Qu}, \mathrm{X}$. et al. Promotion of tumorigenesis by heterozygous disruption of the beclin 1 autophagy gene. J. Clin. Invest. 112, 1809-1820 (2003).

7. Kondo, Y., Kanzawa, T., Sawaya, R. \& Kondo, S. The role of autophagy in cancer development and response to therapy. Nat. Rev. Cancer 5, 726-734 (2005).

8. Guo, J. Y. et al. Activated Ras requires autophagy to maintain oxidative metabolism and tumorigenesis. Genes Dev. 25, 460-470 (2011).

9. Strohecker, A. M. et al. Autophagy sustains mitochondrial glutamine metabolism and growth of BrafV600E-driven lung tumors. Cancer Discov. 3 1272-1285 (2013)

10. Bellodi, C. et al. Targeting autophagy potentiates tyrosine kinase inhibitorinduced cell death in Philadelphia chromosome-positive cells, including primary CML stem cells. J. Clin. Invest. 119, 1109-1123 (2009).

11. Altman, B. J. et al. Autophagy is essential to suppress cell stress and to allow BCR-Abl-mediated leukemogenesis. Oncogene 30, 1855-1867 (2011).

12. Nencioni, A. et al. Autophagy in blood cancers: biological role and therapeutic implications. Haematologica 98, 1335-1343 (2013).

13. Sumitomo, Y. et al. Cytoprotective autophagy maintains leukemia-initiating cells in murine myeloid leukemia. Blood 128, 1614-1624 (2016).

14. Piya, S. et al. Atg7 suppression enhances chemotherapeutic agent sensitivity and overcomes stroma-mediated chemoresistance in acute myeloid leukemia. Blood 128, 1260-1269 (2016).

15. Folkerts, H. et al. Inhibition of autophagy as a treatment strategy for p53 wildtype acute myeloid leukemia. Cell Death Dis. 8, e2927 (2017). 13.

16. Heydt, Q. et al. Oncogenic FLT3-ITD supports autophagy via ATF4 in acute myeloid leukemia. Oncogene 37, 787-797 (2018).

17. Altman, J. K. et al. Autophagy is a survival mechanism of acute myelogenous leukemia precursors during dual mTORC2/mTORC1 targeting. Clin. Cancer Res. J. Am. Assoc. Cancer Res. 20, 2400-2409 (2014).

18. Torgersen, M. L., Engedal, N., Bøe, S.-O., Hokland, P. \& Simonsen, A. Targeting autophagy potentiates the apoptotic effect of histone deacetylase inhibitors in t(8;21) AML cells. Blood 122, 2467-2476 (2013).

19. Larrue, C. et al. Proteasome inhibitors induce FLT3-ITD degradation through autophagy in AML cells. Blood 127, 882-892 (2016).

20. Isakson, P., Bjørås, M., Bøe, S. O. \& Simonsen, A. Autophagy contributes to therapy-induced degradation of the PML/RARA oncoprotein. Blood 116, 2324-2331 (2010).

21. Malaise, M., Steinbach, D. \& Corbacioglu, S. Clinical implications of c-Kit mutations in acute myelogenous leukemia. Curr. Hematol. Malig. Rep. 4, 77-82 (2009).

22. Paschka, P. Adverse prognostic significance of KIT mutations in adult acute myeloid leukemia with inv(16) and t(8;21): A Cancer and Leukemia Group B Study. J. Clin. Oncol. 24, 3904-3911 (2006).

23. Wang, Y.-Y. et al. C-KIT mutation cooperates with full-length AML1-ETO to induce acute myeloid leukemia in mice. Proc. Natl Acad. Sci. USA 108, 2450-2455 (2011).

24. Kabeya, Y. et al. LC3, a mammalian homologue of yeast Apg8p, is localized in autophagosome membranes after processing. EMBO J. 19, 5720-5728 (2000). 
25. Dauer, D. J. et al. Stat3 regulates genes common to both wound healing and cancer. Oncogene 24, 3397-3408 (2005).

26. Scuto, A. et al. STAT3 inhibition is a therapeutic strategy for ABC-like diffuse large B-cell lymphoma. Cancer Res. 71, 3182-3188 (2011).

27. Spitzner, M. et al. STAT3 inhibition sensitizes colorectal cancer to chemoradiotherapy in vitro and in vivo. Int J. Cancer 134, 997-1007 (2014).

28. Karsli-Uzunbas, G. et al. Autophagy is required for glucose homeostasis and lung tumor maintenance. Cancer Discov. 4, 914-927 (2014).

29. Xie, X., Koh, J. Y., Price, S., White, E. \& Mehnert, J. M. Atg7 overcomes senescence and promotes growth of BrafV600E-driven melanoma. Cancer Discov. 5, 410-423 (2015)

30. Galluzzi, L., Pietrocola, F., Levine, B. \& Kroemer, G. Metabolic control of autophagy. Cell 159, 1263-1276 (2014).

31. Willems, L. et al. Inhibiting glutamine uptake represents an attractive new strategy for treating acute myeloid leukemia. Blood 122, 3521-3532 (2013).

32. Jacque, $\mathrm{N}$. et al. Targeting glutaminolysis has antileukemic activity in acute myeloid leukemia and synergizes with BCL-2 inhibition. Blood 126, 1346-1356 (2015).

33. Samudio, I. et al. Pharmacologic inhibition of fatty acid oxidation sensitizes human leukemia cells to apoptosis induction. J. Clin. Invest. 120, 142-156 (2010).

34. Ye, H. et al. Leukemic stem cells evade chemotherapy by metabolic adaptation to an adipose tissue niche. Cell. Stem Cell. 19, 23-37 (2016). 07.

35. Farge, T. et al. Chemotherapy-Resistant Human Acute Myeloid Leukemia Cells Are Not Enriched for Leukemic Stem Cells but Require Oxidative Metabolism. Cancer Discov. 7, 716-735 (2017).

36. Dowdle, W. E. et al. Selective VPS34 inhibitor blocks autophagy and uncovers a role for NCOA4 in ferritin degradation and iron homeostasis in vivo. Nat. Cell Biol. 16, 1069-1079 (2014).

37. Egan, D. F. et al. Small molecule inhibition of the autophagy kinase ULK1 and identification of ULK1 substrates. Mol. Cell. 59, 285-297 (2015).

38. Ronan, B. et al. A highly potent and selective Vps34 inhibitor alters vesicle trafficking and autophagy. Nat. Chem. Biol. 10, 1013-1019 (2014).

39. Mitou, G. et al. Targeting autophagy enhances the anti-tumoral action of crizotinib in ALK-positive anaplastic large cell lymphoma. Oncotarget 6, 30149-30164 (2015).

40. Gotlib, J. et al. Efficacy and safety of midostaurin in advanced systemic mastocytosis. N. Engl. J. Med. 374, 2530-2541 (2016).

41. You, L. et al. The role of STAT3 in autophagy. Autophagy 11, 729-739 (2015).
42. Shen, S. et al. Cytoplasmic STAT3 represses autophagy by inhibiting PKR activity. Mol. Cell. 48, 667-680 (2012).

43. Wegrzyn, J. et al. Function of mitochondrial Stat3 in cellular respiration. Science 323, 793-797 (2009).

44. Kang, R. et al. The expression of the receptor for advanced glycation endproducts (RAGE) is permissive for early pancreatic neoplasia. Proc. Natl Acad. Sci. USA 109, 7031-7036 (2012).

45. Chung, J., Uchida, E., Grammer, T. C. \& Blenis, J. STAT3 serine phosphorylation by ERK-dependent and -independent pathways negatively modulates its tyrosine phosphorylation. Mol. Cell Biol. 17, 6508-6516 (1997).

46. Lim, C. P. \& Cao, X. Serine phosphorylation and negative regulation of Stat3 by JNK. J. Biol. Chem. 274, 31055-31061 (1999).

47. Zhang, Q. et al. Mitochondrial localized Stat3 promotes breast cancer growth via phosphorylation of serine 727. J. Biol. Chem. 288, 31280-31288 (2013).

48. Feng, Y. et al. Metformin promotes autophagy and apoptosis in esophageal squamous cell carcinoma by downregulating Stat3 signaling. Cell Death Dis. 5, e1088 (2014).

49. Tai, W.-T. et al. Mcl-1-dependent activation of Beclin 1 mediates autophagic cell death induced by sorafenib and SC-59 in hepatocellular carcinoma cells. Cell Death Dis. 4, e485 (2013).

50. Miao, L.-J. et al. Stat3 inhibits Beclin 1 expression through recruitment of HDAC3 in nonsmall cell lung cancer cells. Tumour Biol. J. Int Soc. Oncodev. Biol. Med. 35, 7097-7103 (2014).

51. Jung, J. E. et al. STAT3 is a potential modulator of HIF-1-mediated VEGF expression in human renal carcinoma cells. FASEB J. 19, 1296-1298 (2005).

52. Pratt, J. \& Annabi, B. Induction of autophagy biomarker BNIP3 requires a JAK2/ STAT3 and MT1-MMP signaling interplay in Concanavalin-A-activated U87 glioblastoma cells. Cell Signal. 26, 917-924 (2014).

53. Xue, H. et al. A novel tumor-promoting mechanism of IL6 and the therapeutic efficacy of tocilizumab: Hypoxia-induced IL6 is a potent autophagy initiator in glioblastoma via the p-STAT3-MIR155-3p-CREBRF pathway. Autophagy 12, 1129-1152 (2016).

54. Chaix, A. et al. Mechanisms of STAT protein activation by oncogenic KIT mutants in neoplastic mast cells. J. Biol. Chem. 286, 5956-5966 (2011).

55. Xiang, Z., Kreisel, F., Cain, J., Colson, A. \& Tomasson, M. H. Neoplasia driven by mutant c-KIT is mediated by intracellular, not plasma membrane, receptor signaling. Mol. Cell Biol. 27, 267-282 (2007).

56. Auberger P. \& Puissant A. Autophagy, a key mechanism of oncogenesis and resistance in leukemia. Blood 129, 547-552 (2016). 